\title{
Bioactive Compounds from Morus indica as Inhibitors of Advanced Glycation End Products
}

\author{
S. ANANDAN AND ASNA UROOJ* \\ DOS in Food Science and Nutrition, Manasagangotri, University of Mysore, Mysuru-570 006, India
}

Anandan and Urooj: Morus indica as Inhibitors of Advanced Glycation End Products

\begin{abstract}
The present study aimed at identifying the active antiglycation constituents of G4 variety of Morus indica leaves. The bioactive compounds of G4 were identified and quantified using ultra-performance liquid chromatography/mass spectroscopy, isolated by preparative high performance liquid chromatography and characterized by Fourier-transform infrared spectroscopy, nuclear magnetic resonance and scanning electron microscopy. Based on results of the ultra-performance liquid chromatography/mass spectroscopy, extract with maximum bioactive compounds was chosen to study antiglycation property at different stages. The $80 \%$ methanol extract of Morus indica leaves showed presence of 4 compounds, chlorogenic acid, ferulic acid, rutin and apigenin of which apigenin showed the maximum yield. This is the first report on the isolation of apigenin from the leaves of Morus indica G4 variety. Results obtained indicated that Morus indica leaf extract inhibited formation of advanced glycation end products. Also, scanning electron microscopy images indicated the protective effect against the formation of acanthocytes. These results provided evidence for the antiglycation effects of Morus indica leaves and suggested a potential role in management of diabetic complications.
\end{abstract}

Key words: Morus indica, Moraceae, $\delta$-gluconolactone, bovine serum albumin, methylglyoxal, $\mathrm{N}$-acetylglycyllysine methyl ester (GK) peptide

The formation of advanced glycation end products (AGEs) plays an important role in the pathogenesis of diabetic complications like nephropathy, neuropathy and retinopathy ${ }^{[1]}$. AGEs are formed as a result of non-enzymatic reaction of sugars with proteins by the Maillard reaction after undergoing multiple steps ${ }^{[2]}$. The Maillard reaction has been described in the early 1900 s when it was noted that amino acids heated in the presence of reducing sugar developed a characteristic yellow brown colour through a series of reactions forming Schiff bases and Amadori product ${ }^{[3]}$. These reaction series is subdivided into three main stages, early, intermediate, and last stage. In the early stage, glucose, fructose, pentoses, galactose, mannose and xylulose react with a free amino group to form an unstable compound, the Schiff base, which undergoes a rearrangement to a more stable product known as Amadori product. In the intermediate stage, the Amadori product degrades to a variety of reactive dicarbonyl compounds such as glyoxal, methylglyoxal (MGO), and deoxy-glucosones via dehydration, oxidation and other chemical reactions. In the last stage of glycation, irreversible compounds called AGEs are

*Address for correspondence

E-mail: asnaurooj@foodsci.uni-mysore.ac.in

March-April 2019

Indian Journal of Pharmaceutical Sciences formed through oxidation, dehydration and cyclization reactions ${ }^{[4]}$. The AGEs are yellow-brown, fluorescent and insoluble adducts that accumulate on long-lived proteins thus impairing their physiological functions.

Aminoguanidine (AG), an inhibitor of advanced glycation reactions in vitro, has been found to inhibit the development of diabetic complications in animal models of diabetes ${ }^{[5]}$. Inhibition of AGEs was used to treat diabetic complications without much success, perhaps due to relatively lower efficacy, poor pharmacokinetics and toxicity ${ }^{[6]}$. Studies reported the use of medicinal plants as antiglycation compounds instead of the standard drug $\mathrm{AG}^{[7]}$.

In our laboratory, various medicinal plants such as Moringa oleifera, Ficus benghalensis and Morus indica (MI) varieties have been screened for proximate

This is an open access article distributed under the terms of the Creative Commons Attribution-NonCommercial-ShareAlike 3.0 License, which allows others to remix, tweak, and build upon the work non-commercially, as long as the author is credited and the new creations are licensed under the identical terms

Accepted 07 February 2019

Revised 10 September 2018

Received 25 January 2018

Indian J Pharm Sci 2019;81(2):282-292 
composition, phytochemical profile, antimutagenic, antioxidant, antihypercholesterolemic, antidiabetic and protease activities in in vitro and ex vivo models. Further, the possible mechanisms of antidiabetic action of F. racemosa bark and MI leaves were elucidated through pre-clinical and clinical studies ${ }^{[8-11]}$. MI is a non-toxic natural therapeutic plant used as herbal medicine as hypoglycaemic and diuretic ${ }^{[12]}$. In our previous studies, on $80 \%$ methanol leaf extract of MI G4 variety (AQME), two C-glycosyl flavonoid compounds, apigenin and luteolin were detected ${ }^{[13]}$. The chemical constituents along with the above mentioned flavonoids of AQME exhibited strong metal-ion chelating and inhibitory effects of AGEs (bovine serum albumin, BSA-glucose model) in vitro. Hence, further studies were planned to conduct inhibition of AGEs at the early, intermediate and final stages. To the best of our knowledge, this is the first study to report the inhibitory effects of AQME at each stage of protein glycation.

\section{MATERIALS AND METHODS}

The leaves of commercial varieties of MI G4 (MIG4, ISGR Reg. No.: 050564), were collected in the month of May-2016 from Central Sericulture Research and Training Institute, Mysore. Bovine serum albumin (purity $>98 \%$ ), MGO $\left(40 \%\right.$ in $\left.\mathrm{H}_{2} \mathrm{O}\right)$, acetylglycyllysine methyl ester (GK) peptide (purity $>98 \%$ ), AG hydrochloride (purity $>98 \%$ ) and high performance liquid chromatography (HPLC) standard chlorogenic acid (purity $>95 \%$ ), ferulic acid (purity $>99 \%$ ), apigenin (purity $>95 \%$ ), myricetin (purity $>96 \%$ ), quercetin (purity $>95 \%$ ), kaempferol (purity $>97 \%$ ), catechin (purity $>98 \%$ ) and rutin (purity $>94 \%$ ) were purchased from Sigma-Aldrich Chemicals Co. (St. Louis, MO, USA). $\delta$-Gluconolactone and Nile red were purchased from HiMedia. All other chemicals used were of analytical-grade.

\section{Extraction and isolation of bioactive components from MIG4 using different solvents:}

Powdered MI was packed in a glass column, retained for $2 \mathrm{~h}$ in hexane and later gradually eluted with hexane till the eluent became colourless. The remaining material after complete removal of hexane was further soaked and eluted with different solvents sequentially with increasing polarity such as chloroform, acetone, ethyl acetate, methanol, aqueous methanol (80\%) and finally with water. Each fraction thus obtained was concentrated under reduced pressure.

\section{Identification and quantification of chemical constituents in MIG4:}

Liquid chromatography-mass spectrometry analysis was performed using a Waters (Acquity UPLC, USA) system coupled to a quadrupole time of flight (Q-TOF) mass spectrometer (Synapt G2, USA) equipped with an electrospray ionization (negative mode) source that was used at an ion source temperature of $100^{\circ}$. The detection and quantification by ultra-performance liquid chromatography/mass spectrometry (UPLC/ MS) method was followed according to the previous reports ${ }^{[14]}$. The test sample was dissolved in $80 \%$ methanol and $2 \mu \mathrm{l}$ of sample was injected on Acquity UPLC BEH C18 column $(1.7 \mu \mathrm{m} 1.0 \times 50 \mathrm{~mm})$ at a flow rate of $0.3 \mathrm{ml} / \mathrm{min}$. Solvent A consisted of $0.1 \%$ formic acid in water ( $\mathrm{pH} 2.75)$ and solvent $\mathrm{B}$ consisted of acetonitrile. Gradient program was used for elution. Initially, the concentration of solvent B was $2 \%$, which was increased to $98 \%$ at $4 \mathrm{~min}$, maintained up to $6 \mathrm{~min}$ and finally at $8 \mathrm{~min}$ the concentration of solvent B was $2 \%$. The mass spectrometer was operated in $[\mathrm{M}-\mathrm{H}]$ negative ion mode. Capillary voltage was set at $2.5 \mathrm{kV}$ and the cone voltage was optimised for each of the compounds. Molecular species were identified within the mass to charge ratio $(\mathrm{m} / \mathrm{z})$ range 50 to 1500 . Data acquisition was carried out by Mass Lynx software (version: 4.1).

\section{Purification of constituents on preparative HPLC:}

The chromatographic separation was carried out on a Shimadzu Prep LC8A Preparative Chromatography system equipped with SCL-10AVP system controller (Shimadzu). The preparative HPLC was performed on a Waters Spheris orb S-10 ODS-2 20×250 mm SemiPrep column. The flow rate was $8 \mathrm{ml} / \mathrm{min}$ and the wavelength used for detection was $320 \mathrm{~nm}$. The solvent system employed was $0.1 \%$ formic acid in water and acetonitrile (80:20). The sample volume injected was $2 \mathrm{ml}$. Peaks were collected manually, concentrated and evaporated to dryness by rotary evaporator.

\section{Fourier-transform infrared (FTIR) spectroscopy:}

Functional groups in the isolated compound were detected by FTIR analysis. The characterization involved FTIR analysis of the dried powder of the isolated compound by PerkinElmer Spectrum Version 10.03.09 (PerkinElmer, Inc., USA) in attenuated total reflection mode using the spectral range of 4000$400 \mathrm{~cm}^{-1}$ with a resolution of $4 \mathrm{~cm}^{-1}$ 
Nuclear magnetic resonance (NMR) analysis:

${ }^{1} \mathrm{H}$ and ${ }^{13} \mathrm{C}$ NMR spectra for the compounds were recorded on a Bruker Avance $500 \mathrm{MHz}$ spectrometer (Bruker biospin, Reinstetten, Germany) using dimethyl sulfoxide (DMSO) solvent.

\section{Morphological analysis by scanning electron microscopy (SEM):}

Morphological features of the isolated compound were determined by SEM (Zeiss, Germany) at $15 \mathrm{kV}$ and under low vacuum at 45 Pascal. The images were taken at magnifications of 500X, 2000X and 5000X.

\section{Haemoglobin- $\delta$-gluconolactone (HDG) assay:}

As reported earlier, the method to evaluate the inhibitory effect of MIG4 on early stage of protein glycation product (Amadori) formation assay was carried out by incubating $200 \mu 1$ of fresh human blood with $\delta$-gluconolactone $(\delta \text {-glu, } 50 \mathrm{mM})^{[15]}$. Test samples at three different concentrations (100$300 \mu \mathrm{g})$ of MIG4 along with control set, which included blood + phosphate-buffered saline (PBS, $\mathrm{pH}$ $7.4)$ as a control, blood $+\delta$-glu (glycated) and blood $+\delta$ glu $+\mathrm{AG}$ (positive control) were evaluated. After incubation, percent hemoglobin $\mathrm{A} 1 \mathrm{C}$ (HbA1C) content in the samples was estimated using haemoglobin $\mathrm{A} 1 \mathrm{C}$ chromatographic-spectrophotometric ion exchange kit (Biosystems, India).

\section{Sample preparation for intermediate stage of glycation:}

BSA was glycated using MGO as a glycating agent ${ }^{[16]}$. The reaction mixtures contained BSA $(10 \mathrm{mg} / \mathrm{ml})$ with MGO $(10 \mathrm{mM})$ in a final volume of $1 \mathrm{ml}$ of $0.1 \mathrm{M}$ PBS (pH 7.4). The reaction mixture was incubated at $37^{\circ}$ for $7 \mathrm{~d}$ in the presence of different concentrations of MIG4 $(100,200$ and $300 \mu \mathrm{g} / \mathrm{ml})$. BSA-MGO with or without AG $(10 \mathrm{mM})$ served as positive and negative control, respectively while only BSA served as native. After incubation, modified BSA was extensively dialyzed using dialysis membrane-110 (HiMedia, India) having molecular weight cut off between 12000 to 14000 against PBS for $24 \mathrm{~h}$ to remove unbound MGO and was stored in the deep freezer $\left(-20^{\circ}\right)$ until further use.

\section{Protein glycation and aggregation of BSA-MGO:}

The MIG4 (100, 200 and $300 \mu \mathrm{g} / \mathrm{ml})$ was evaluated for the formation of total AGEs by their characteristic fluorescence at $330 \mathrm{~nm}$ (excitation) and $440 \mathrm{~nm}$ (emission). The effect of MGO modification on the cross-linking and aggregation of BSA was also investigated by sodium dodecyl sulphatepolyacrylamide gel electrophoresis (SDS-PAGE) as described ${ }^{[17]}$ under reducing conditions (4\% stacking and $10 \%$ separating gel). Thioflavin-T (ThT) fluorescence assay was carried out to know the aggregation of BSA-MGO according to method ${ }^{[18]}$. Fluorescence measurements were carried out at excitation (435 nm) and emission (485) wavelengths. FTIR spectroscopy was used to investigated with slight modification the changes in structure in BSA-MGO upon treatments ${ }^{[19]}$.

\section{Fluorescence microscopy:}

Nile red $(25 \mu \mathrm{M})$ and of ThT $(1 \mathrm{mM})$ dye solutions were freshly prepared in phosphate buffer $(20 \mathrm{mM}$, $\mathrm{pH}$ 7.4) ${ }^{[20]}$. The dye solution was immediately added to the samples with the final concentration of $5 \mu \mathrm{M}$ of Nile red and $100 \mu \mathrm{M}$ of ThT. About $10 \mu \mathrm{l}$ aliquot of the samples pre-stained with Nile red and ThT were placed on glass slides and images were taken using a fluorescence microscope (Imager A2, Axico Cam MRc5, Zeiuss).

\section{Protective effect of MIG4 on red blood cells (RBCs):}

Blood sample $(2 \mathrm{ml})$ was collected from a healthy individual in citrate-containing tubes. The sample preparation was done as described earlier ${ }^{[21]}$. RBCs were separated by centrifuging the blood sample at $1000 \mathrm{rpm}$ for $20 \mathrm{~min}$. After centrifugation, white blood cells and other plasma proteins were decanted. The pellet containing RBCs was washed thrice with PBS. These cells were then added with MGO $(5 \mu \mathrm{M})$ in the presence and absence of $200 \mu \mathrm{g}$ MIG4 and allowed to react for $1 \mathrm{~h}$. Then, the reaction mixture was centrifuged to pellet the RBCs and washed thrice with PBS. Then, the cells were subsequently fixed with $2.5 \%$ glutaraldehyde for $30 \mathrm{~min}$, which was followed by centrifugation (1000 rpm, $30 \mathrm{~min}$ ) to remove the glutaraldehyde and washed thrice with PBS. Then $5 \mu$ of fine suspension of RBCs were placed on an aluminium foil and allowed to dry overnight. It was furthermore coated with gold and analysed using SEM under high vacuum at 72 Pascal (Hitachi S-3400N, Japan).

\section{N-Acetylglycyl-lysine methyl ester (GK) peptide- ribose assay (last stage glycation):}

This test was used to evaluate the ability of MIG4 at $200 \mu \mathrm{g}$ concentration to inhibit the cross-linking of GK peptide $(80 \mathrm{mg} / \mathrm{ml})$ in the presence of ribose $(0.8 \mathrm{M})$ according to the method described ${ }^{[22]}$ under 
sterile conditions in $0.5 \mathrm{M}$ sodium phosphate buffer $(\mathrm{pH}$ 7.4 ) at $37^{\circ}$ for $24 \mathrm{~h}$. At the end of the incubation period, samples will be analysed for specific fluorescence (excitation $(340 \mathrm{~nm})$ and emission $(420 \mathrm{~nm})$ ).

\section{Statistical analysis:}

Data from three replicates of each experiment was subjected to analysis of variance using SPSS Inc. 16.0. Significant effects of treatments were determined by $\mathrm{F}$ values $(p \leq 0.05)$. Treatment means were separated by Tukey's HSD test.

\section{RESULT AND DISCUSSION}

Chemical constituents in plants exhibit various polarities, thus making their separation and characterization a big challenge. Extraction with suitable solvents is a crucial step in characterization studies. In the present study, different solvents with varying polarities were used. Among all the solvent systems, hexane extract $(7.87 \%)$ yielded maximum solids followed by methanol $(7.37 \%), 80 \%$ methanol (4.25\%), water $(4.15 \%), 80 \%$ chloroform $(1.6 \%)$, acetone $(1.3 \%)$ and ethyl acetate $(0.25 \%)$. Further, all the extracts were subjected to UPLC/MS for bioactive identification and quantification analysis as shown in fig. 1. The results of sequential extraction showed absence of all the standard compounds in hexane, chloroform and acetone solvents. The compounds were

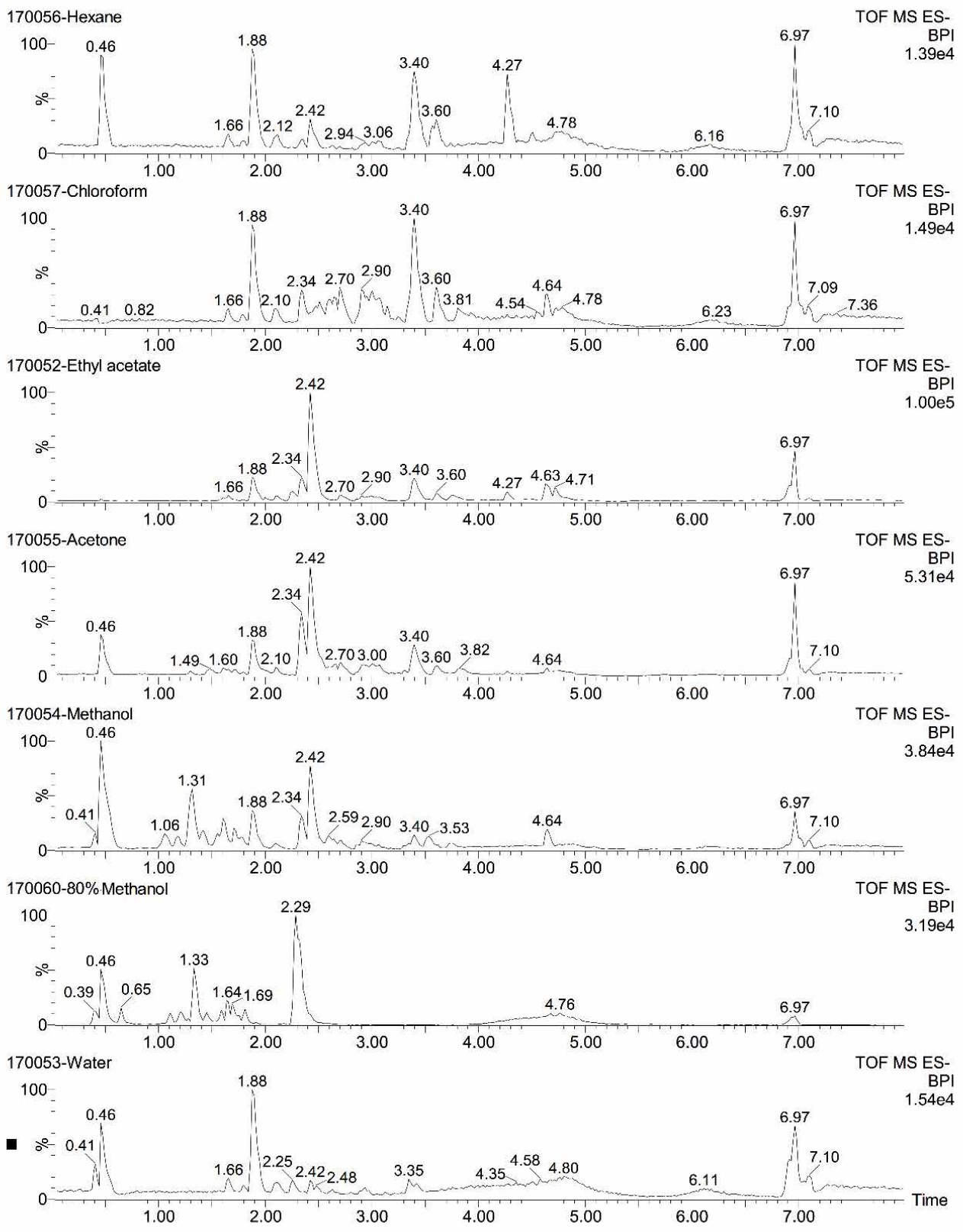

Fig. 1: Chromatogram of sequential extracts of MIG4 by UPLC-MS 
detected in ethyl acetate, methanol, $80 \%$ methanol and aqueous solvents. Among the solvents, compounds were highly soluble and detected in $80 \%$ methanol followed by methanol, ethyl acetate and aqueous solvents. The $80 \%$ methanol extract showed presence of 4 compounds viz., chlorogenic acid, ferulic acid, rutin and apigenin. Apigenin was detected at a higher level when compared to other solvents as shown in Table 1. Since the primary focus of the study is related to the role of bioactive compounds in inhibition of different stages of AGEs, apigenin was considered for further isolation and purification.

The compound purified by preparative HPLC from $80 \%$ methanol extracts of MIG4 was subjected to UPLC/MS analysis in negative ionization mode. The chromatogram and mass spectra of MIG4 matched with the standard apigenin as shown in fig. 2. The mass spectra of standard apigenin and MIG4 variety showed a characteristic peak in negative ion mode showing retention time 2.253; [M-H] $]^{-}, \mathrm{m} / \mathrm{z} 268.9872$ (fig. 2A and $\mathrm{B}$ ) and 2.270; [M-H]', $m / z 268.9$ (fig. 2C and D, respectively). This is the first report with respect to the presence of C-glycosyl flavonoids of apigenin in MIG4 variety.

The results of FTIR showed intensive bands between 1605.44 and $1580 \mathrm{~cm}^{-1}$, which corresponded to aromatic ring vibrations. The bands at 3292.39 and $3097.46 \mathrm{~cm}^{-1}$ probably were the result of hydrogen bonded $\mathrm{O}=\mathrm{H}$ stretch and $\mathrm{C}-\mathrm{H}$ vibrations before respectively. The bands between 1297.65 and $1004.38 \mathrm{~cm}^{-1}$ corresponded to $\mathrm{C}-\mathrm{O}$ stretch vibrations. The intensive bands between 1444.15 and $1497.98 \mathrm{~cm}^{-1}$ were assigned as $\mathrm{C}-\mathrm{H}$ vibrations and the peak at $2621 \mathrm{~cm}^{-1}$ was assigned to the $\mathrm{C}-\mathrm{H}$ symmetric stretching. With these above spectral characteristics, it is indicated that the probable compound is apigenin as shown in fig. 3A.

In NMR analysis, sample obtained was a yellow amorphous powder, EI-MS $m / z$ : $[\mathrm{M}+\mathrm{H}]+268.8$, which matched the molecular formula $\mathrm{C}_{15} \mathrm{H}_{10} \mathrm{O}_{5} ;{ }^{1} \mathrm{H}$ NMR $\left(400 \mathrm{MHz}, \mathrm{DMSO}, \mathrm{d}^{6}\right): \delta 12.933-1 \mathrm{H}(\mathrm{s}, \mathrm{OH})$ at C-3', $10.782-1 \mathrm{H}(\mathrm{s}, \mathrm{OH})$ at $\mathrm{C}-4,10.311-1 \mathrm{H}(\mathrm{s}, \mathrm{OH})$ at $\mathrm{C}-2$, 7.907- $\left.2 \mathrm{H} \mathrm{(d,} \mathrm{C-2'} \mathrm{and} \mathrm{C} 4{ }^{\prime}\right), 6.906-2 \mathrm{H}\left(\mathrm{d}, \mathrm{C}-1^{\prime}\right.$ and C-5'), 6.750-1H (s, C-1), 6.454- 1H (s, C-3), 6.165-1H (s, C-8). ${ }^{13} \mathrm{C}$ NMR (400 MHz, DMSO, d ${ }^{6}$ ) d 182.177(C-9), 164.571- (C-2), 164.200- (C-4), 161.906(C-7), 161.611- (C-6), 157.766- (C-3'), 128.904(C-5') and (C-1') 121.644- (C-6'), 116.408- (C-4') and (C-2'), 104.157- (C-5), 103.305- (C-8) 99.283- (C-3), 94.401- (C-1). Thus, according to the mentioned data and by comparison with the literature, compound was confirmed as apigenin as shown in fig. 3B.

The morphology of isolated compound apigenin from MIG4 was studied by SEM under low vacuum at 45 Pascal. Isolated compound showed a surface

TABLE 1: CONTENT OF BIOACTIVE COMPOUNDS OF MIG4 IN SEQUENTIAL EXTRACTS BY UPLC-MS

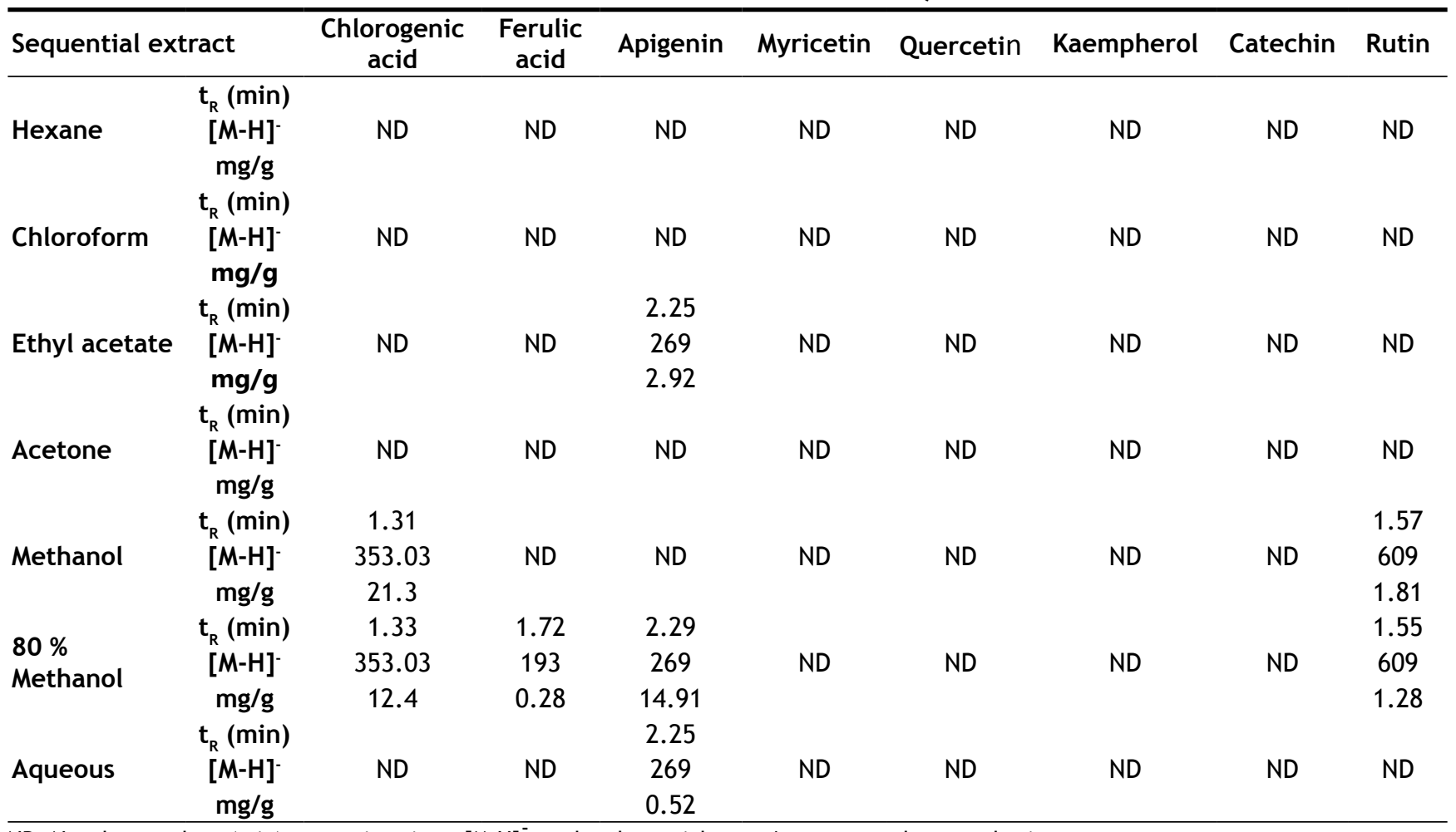

ND: Not detected; $t_{R}(\mathrm{~min})$ : retention time; $[\mathrm{M}-\mathrm{H}]^{-}$: molecular weight; $\mathrm{mg} / \mathrm{g}$ : expressed on wet basis 

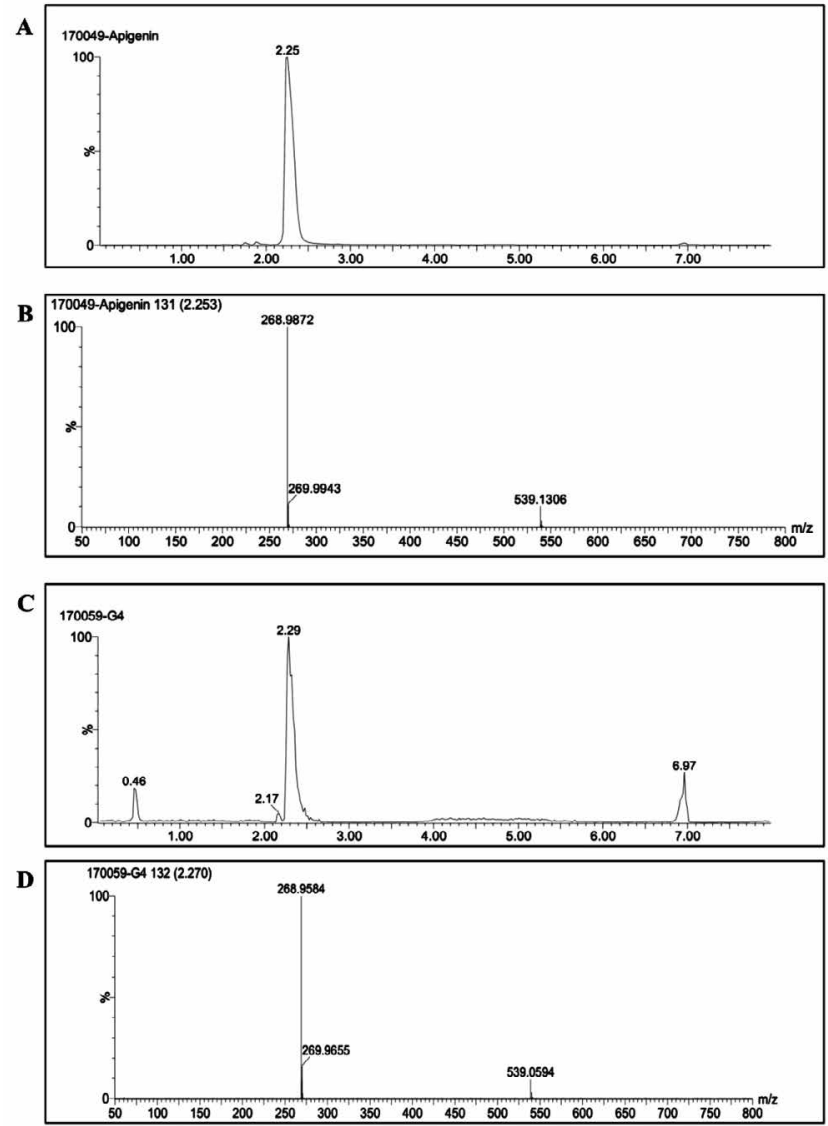

Fig. 2: Isolated bioactive compound in MIG4 by UPLC/MS

(A) Chromatogram of standard apigenin showing retention time 2.25; (B) mass spectra of standard apigenin $\left([\mathrm{M}-\mathrm{H}]^{-}, \mathrm{m} / \mathrm{z}\right.$ 268.9872); (C) chromatogram of MIG4 showing apigenin peak at retention time 2.29; (D) mass spectra of MIG4 apigenin $\left([\mathrm{M}-\mathrm{H}]^{-}, m / z, 268.958\right.$ with irregular spiral shape. The images were taken at magnifications of 500, 2000 and 5000X as shown in the fig. $3 \mathrm{C}$ to $\mathrm{E}$.

In general, the mechanism of formation of AGEs includes the following stages. In the initial stage, glucose reacts with an amine group to form a labile Schiff base that rearranges to form Amadori product. The Schiff base is highly prone to oxidation and free radical generation, which leads to the formation of reactive carbonyl species (RCS) such as glyoxal and the last stage is formation of AGEs and cross-linking with other compounds ${ }^{[23]}$. In this study, the inhibition of AGEs was demonstrated in the reaction mixtures containing different amines and different sugar sources. Sugar sources ( $\delta$-Glu, MGO and ribose) were used as glycating agents, which are commonly adopted in many Maillard reaction studies. Amine sources (human haemoglobin, BSA and GK peptide) served as targets for glycating agent. These experimental methods can uniquely differentiate between specific inhibitors of the early stage (Amadori products), intermediates stage (RCS), and the last stage of glycation (AGEs formation and cross-linking). The degrees of glycation were analysed for the development of specific fluorescence, and $\mathrm{AG}$ was used as a positive control ${ }^{[24]}$. Though apigenin was identified, isolated and characterized, $80 \%$ methanol extract containing four known bioactive compounds was chosen to study the inhibitory potential of bioactives against different stages of

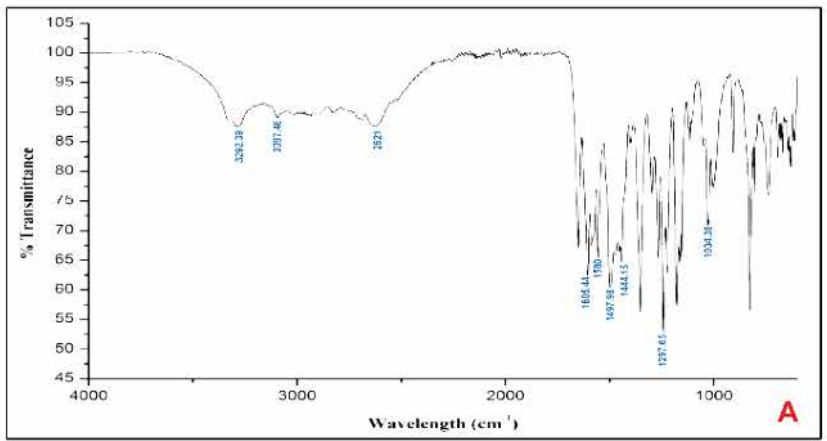<smiles>O=c1cc(-c2ccc(O)cc2)oc2cc(O)cc(O)c12</smiles>
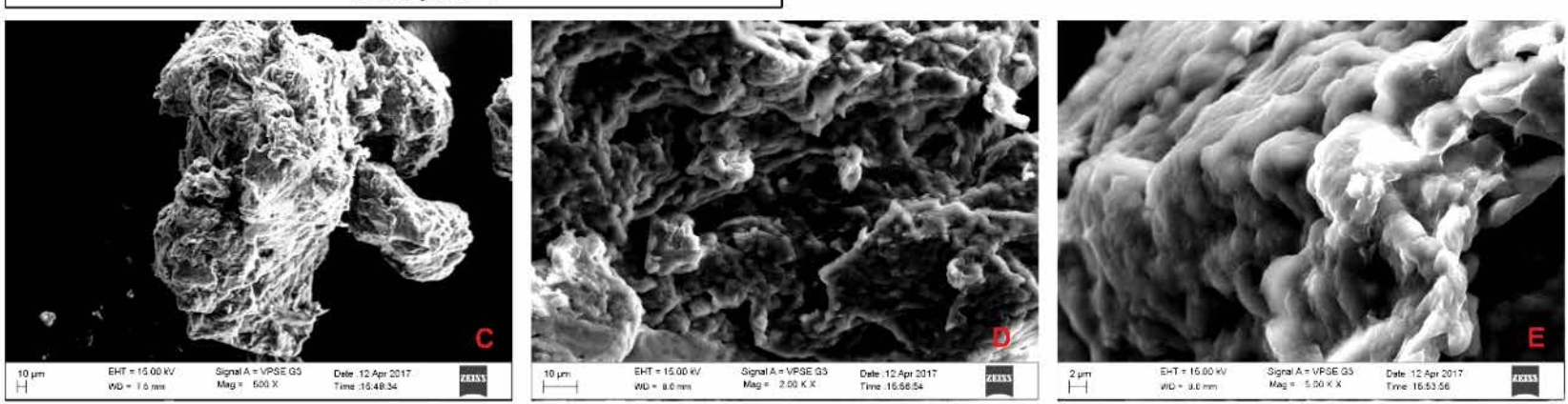

Fig. 3: Identification and characterization of isolated bioactive compound

(A) FTIR profiles of functional groups of apigenin of MIG4; (B) NMR structure of apigenin of MIG4; (C, D and E) SEM images of apigenin of MIG4 at magnification of 500, 2000 and 5000X, respectively 
AGEs formation. Many studies supported the fact that bioactives in combination have better biological activity than one single compound ${ }^{[25,26]}$ and the action was also observed in $80 \%$ methanol extract ${ }^{[27]}$. Hence, further studies were carried out in $80 \%$ methanol extract to evaluate the different stages of protein glycation.

HDG system is used to identify the early stage (Amadori product formation) of glycation. $\delta$-Gluconolactone, an oxidized (ketoaldehyde) analogue of glucose reacts with haemoglobin to form an Amadori product, HbAlc. In our study, early stage of glycation or Amadori product formation was measured by using ion-exchange HPLC (Biosytems). In HDG system, the absence or presence of different amounts of MIG4 extract are shown in fig. 4. After incubation, the content of glycated haemoglobin was significantly higher in HDG reaction mixture in comparison with the control set containing only haemoglobin (fresh blood). However, glycated haemoglobin level decreased gradually in a dose-dependent manner in the presence of MIG4 extract in HDG reaction mixture. This suggests that MIG4 has a greater role in the inhibition of the early AGEs formation. The inhibition exhibited by MIG4 is attributed to its antioxidant capacity. During early stage (Amadori) of glycation the formation of free radicals occurs ${ }^{[28]}$. MIG4 might have larger impact in the inhibition of glycated analogue of haemoglobin, as observed by its antioxidant capacity.

BSA-MGO system is in use for studying inhibition of intermediates stage of glycation. In the intermediates stage of protein glycation, Amadori products form

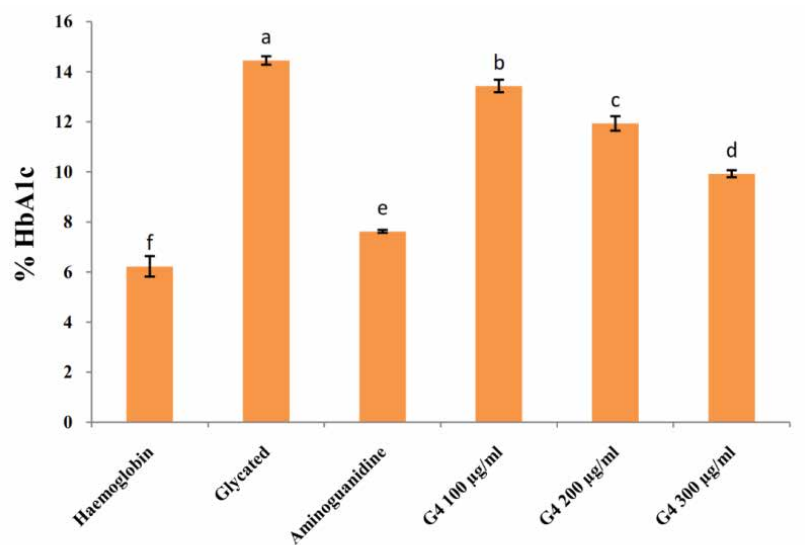

Fig. 4: Early stage of glycation by haemoglobin- $\delta$-gluconolactone assay

Test samples at three different concentrations (100-300 $\mu \mathrm{g})$ of MIG4 along with control set, which includes blood+PBS (pH 7.4, haemoglobin), blood $+\delta$-glu (glycated) and blood $+\delta$ glu + AG (positive control). Results are means \pm SD for $n=3$. $\mathbf{p}<0.05$ compared with haemoglobin (unglycated). Groups with different letters superscripts are significantly different $(p<0.05)$
RCS, namely MGO, glyoxal, which, in turn, react with proteins. Reaction of a dicarbonyl compound with proteins thus mimics intermediate stage of protein glycation. BSA-MGO glycation model was used in order to determine the antiglycation potential of MIG4 towards MGO-mediated glycation. Results of BSAMGO assay are shown in the fig. 5. MIG4 showed inhibition of MGO-mediated glycation of albumin, which was evident from the low fluorescence intensity as observed in the case of test samples when compared to the control as shown in fig. 5A. To understand the MGO-mediated changes in the protein and the effect of MIG4 in the intervention of glycation SDSPAGE was performed for BSA as shown in fig. 5B. In comparison to the blank set containing only BSA (lane 1), BSA-MGO incubation mixture exhibited proteolytic cleavage (lane 2), indicating glycationinduced oxidative cleavage of the protein. As the concentration of MIG4 was increased, the proteolytic cleavage appeared to be progressively inhibited (lanes 4 to 6). These results showed that MIG4 exhibit potent antiglycation activity against MGO modification of albumin. The inhibitory activity of MIG4 for MGO glycation of albumin could be attributed to its carbonyl scavenging potential, which needs to be studied further.

The results were similar to previous studies reported on glucose-treated $\mathrm{BSA}^{[13]}$. The literature also supported these findings in the use of natural flavonoids (luteolin, rutin, epigallocatechin gallate, and quercetin) against all stages of AGEs formation hypoglycaemic and hypolipidemic activity of mulberry (MI) in type 2 diabetes patients ${ }^{[24]}$. ThT binding was used in order to investigate the preventive effect of MIG4 on the post glycation aggregation of albumin. The results for the ThT binding are shown in the fig. 5C. Glycated albumin showed maximum ThT binding, which was evident from high ThT fluorescence intensity when compared to the MIG4 samples. The presence of different concentration (100-300 $\mu \mathrm{g})$ of MIG4 resulted in comparatively low ThT binding and reduced fluorescence intensity. Results suggested that MIG4 prevented the glycationinduced aggregation of albumin. ThT exhibits high specificity to the amyloid aggregates and frequently used to detect amyloid aggregation of proteins. The mechanism of ThT binding to amyloid aggregates has been defined, which suggests that ThT bind to the beta sheet with high affinity ${ }^{[29]}$. The reduced ThT binding was seen for the samples glycated in the presence of MIG4 suggesting its protective effects on aggregation and fibrillation of albumin. MIG4 was found to protect 
the native albumin conformation through hydrophobic interactions. The change in peak positions in the amide I and II regions and transmittance in FTIR spectroscopy demonstrated a change in the secondary structure of BSA after modification with MGO. FTIR spectra of BSA-MGO mixtures incubated for $7 \mathrm{~d}$ with different concentration of MIG4 (100-300 $\mu \mathrm{g})$ were analysed in the range of $1400-1700 \mathrm{~cm}^{-1}$ as shown in fig. 5D. Secondary structure analysis was based on amide-I and amide-II bands within the $1400-2000 \mathrm{~cm}^{-1}$ region after subtraction of the background absorption. In the IR region, the amide I peak position occurred in the $1600-1700 \mathrm{~cm}^{-1}$ region (mainly $\mathrm{C}=\mathrm{O}$ stretch), while the amide II band was located from 1500$1600 \mathrm{~cm}^{-1}(\mathrm{C}-\mathrm{N}$ stretch coupled with $\mathrm{N}-\mathrm{H}$ bending mode), representing the amount of carbonyl and amino bonds in side chains of amino acid residues of the BSA structure $^{[30]}$. In the present, study native BSA showed spectra at 1645.48 and $1547.0 \mathrm{~cm}^{-1}$ in the amide I and II region, respectively. The change in band position and increase in transmission in BSA-MGO was due to the BSA reaction with $\mathrm{MGO}$ as shown in fig. 5D, which indicated that the altered secondary structural elements evolved due to the glycation reaction. As in the presence of MIG4 in BSA-MGO solution, transmission and band positions (amide I and II) showed minimal changes, indicating more unaffected amide bonds and amino groups in the presence of MIG4. However, the effects of MGO on BSA secondary structure were found to gradually decrease with increasing concentrations of MIG4, indicating a dose-dependent effect. The FTIR analysis of the BSA-MGO mixture with and without MIG4 corroborate that MIG4 plays an important role in maintaining the secondary structure of BSA protein.

Glycation-induced conformational changes are known to stimulate aggregation and fibrillation of globular proteins such as haemoglobin and albumin. Fluorescence microscopic imaging was carried out in order to gain insights into the inhibition of aggregation of albumin by MIG4. NR and ThT binds with the amyloid aggregates and exhibits red fluorescence and green blue fluorescence, respectively. Results of fluorescence imaging are shown in the fig. 6. In the present study, control BSA showed less intense fluorescence images compared to BSA incubated with $\mathrm{MGO}$, which is due to glycation of BSA. However,

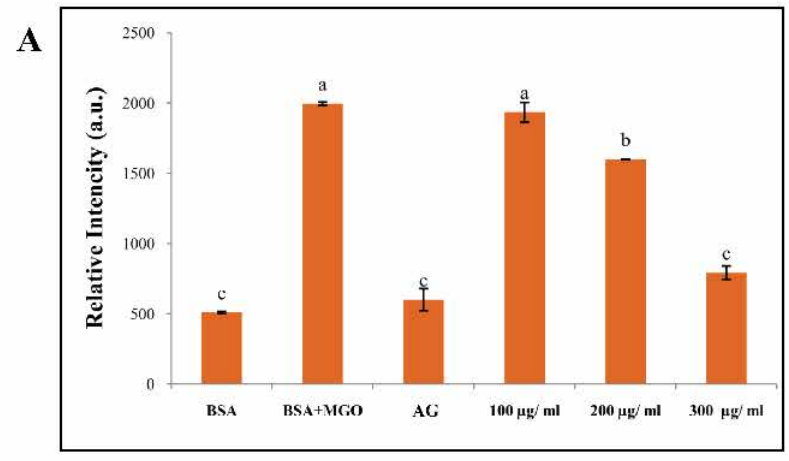

B
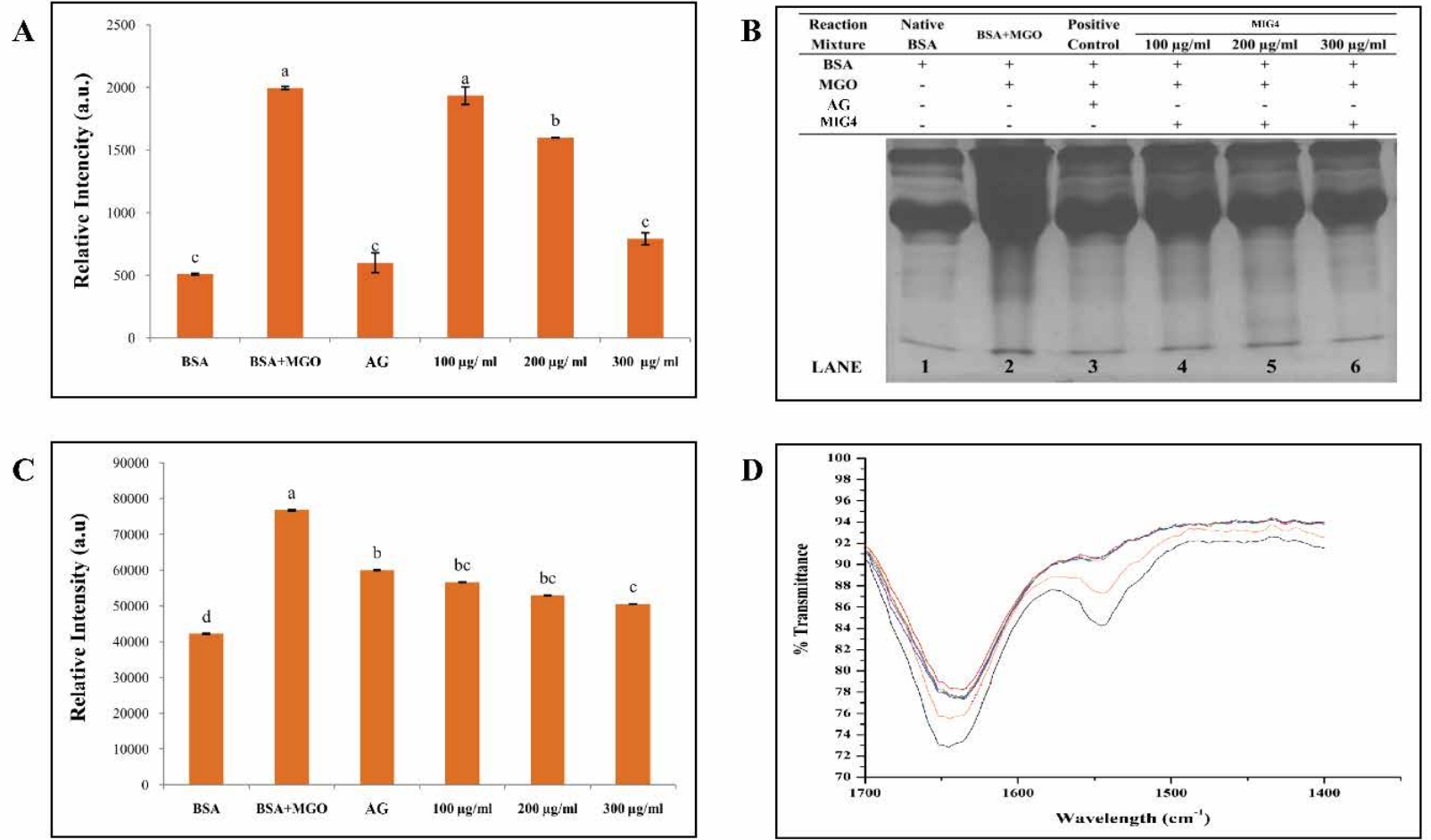

Fig. 5: Intermediate stage of protein glycation and aggregation of BSA-MGO

(A) Antiglycative capacity of MIG4 in the BSA-MGO assay at $37^{\circ}$ for 7 days following analysis of fluorescent AGEs (B) SDS-PAGE profile: lane 1, native BSA; lane 2, control; lane 3, positive control (AG); lane 4, MIG4 extract $100 \mu \mathrm{g} / \mathrm{ml}$; lane 5, MIG4 extract 200 $\mu \mathrm{g} / \mathrm{ml}$; lane $6 \mathrm{MIG} 4$ extract $300 \mu \mathrm{g} / \mathrm{ml}$. (C) ThT binding to the aggregated protein. The fluorescence of ThT is recorded with Ex 435 $\mathrm{nm}$ and Em $485 \mathrm{~nm}$, respectively. (D) FTIR profiles of amide I and amide II bands of BSA and MGO mixtures with and without MIG4 after 7 days of incubation, - BSA, - BSA+MGO, - AG $,-100 \mu \mathrm{g} / \mathrm{ml},-200 \mu \mathrm{g} / \mathrm{ml},-300 \mu \mathrm{g} / \mathrm{ml}$. Results are means \pm SD for $n=3$. $p<0.05$ compared with BSA (unglycated). Groups with different letters superscripts are significantly different ( $<<0.05$ ) 


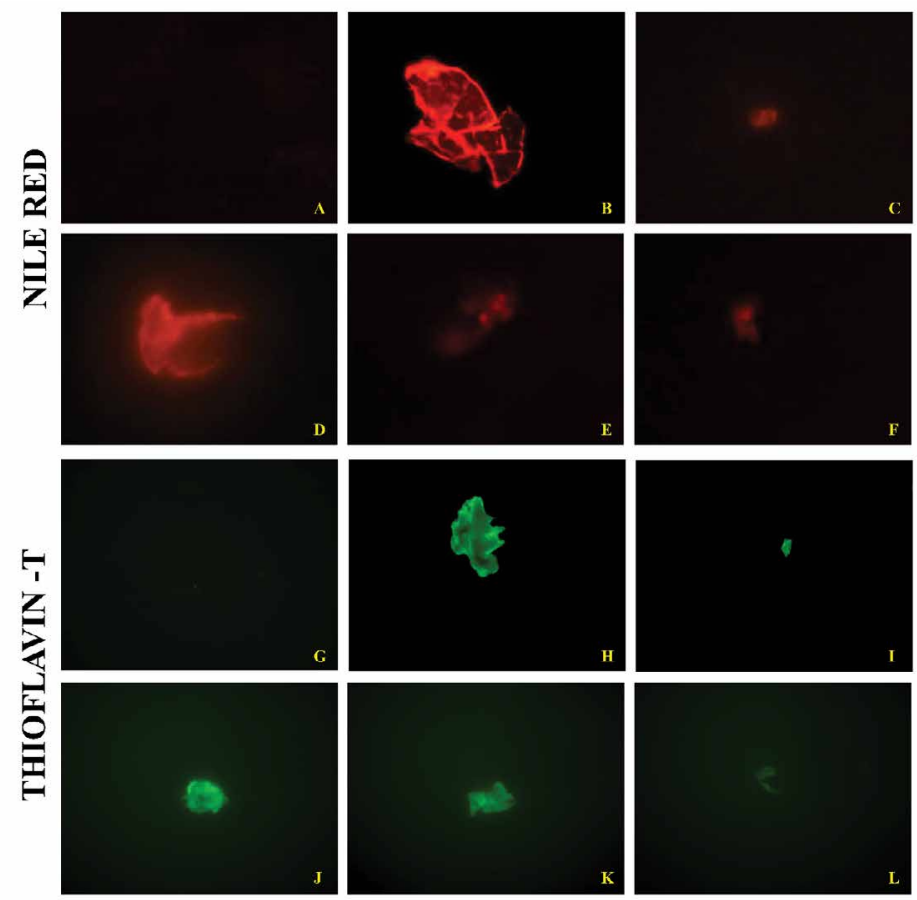

Fig. 6: Nile red and Thioflavin-T photograph by using fluorescence microscopy

BSA incubated with MGO and different concentration of MIG4 extract in 0.1 M phosphate buffer saline (pH 7.4) at 37 for 7 days. (A and G) BSA; (B and H) control; (C and I) AG $10 \mathrm{mM}$; (D and J) MIG4 extract $100 \mu \mathrm{g}$; (E and K) MIG4 extract $200 \mu \mathrm{g}$; (F and

L) MIG4 extract $300 \mu \mathrm{g}$
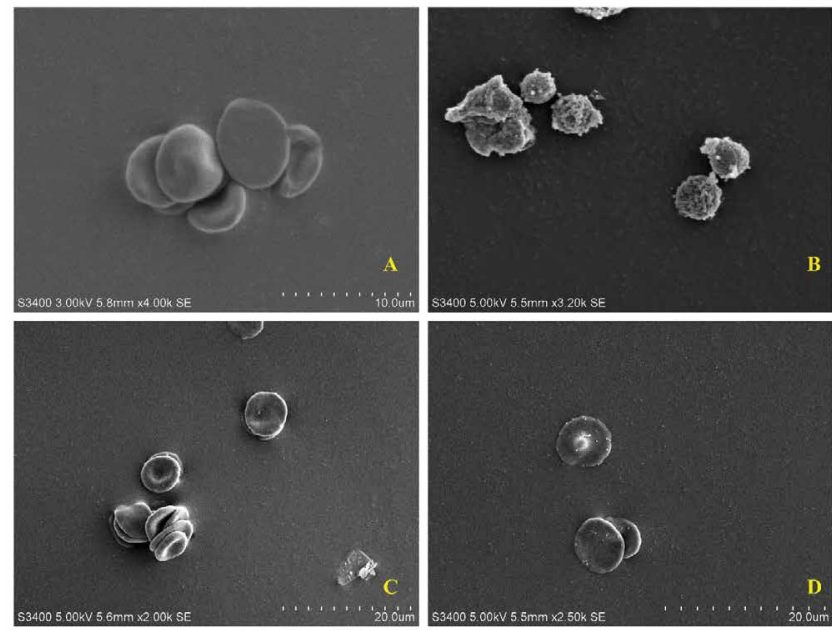

Fig. 7: Protective effect of MIG4 on RBCs

(A) Normal RBCs showing typical biconcave shaped. (B) MGO on RBCs showing the loss of biconcave shape. (C) MGO on RBCs in the presence of $A G$ (D) MGO on RBCs in the presence of $200 \mu$ g concentration of MIG4

presence of different concentrations of MIG4 such as 100,200 and $300 \mu \mathrm{g}$ decreased the fluorescence intensity of the protein. The inhibitory potential of MIG4 at 300 $\mu \mathrm{g}$ was comparable to that $10 \mathrm{mM}$ AG (fig. 6). These results were in good agreement with the ThT binding assays and reaffirmed the preventive effect of MIG4 on the glycation-induced aggregation and fibrillation of albumin. In general, the prolonged glycation of BSA can induce refolding with a conformational transition from the native helical structure to $\beta$-sheeted amyloid form. Based on these observations, it is proposed that glycation as a predisposing factor for amyloidosis ${ }^{[31]}$. The preventive effects of MIG4 for glycation-induced aggregation and fibrillation could be attributed to its potent antiglycation effects and AGEs inhibition activity. The reduced AGEs formation in the presence of MIG4 eventually contributed towards the inhibition of post-glycation aggregation of albumin.

MGO is identified as important precursors of AGEs in vivo and can readily bind to amino groups, thereby modifying biological molecules to form covalently cross-linked aggregates. The elevated levels of MGO disrupt the normal function of RBCs and results in the formation of echinocyte. The change in shape of RBCs will eventually lead to the loss of its function of oxygen transport protein haemoglobin, which ultimately cause oxidative stress ${ }^{[32]}$. In the present study, RBCs showed typical biconcave shape in the absence of MGO (fig. 7A). However, in the presence of MGO the biconcave shape of the RBCs were completely lost, twisted, and ruptured (fig. 7B). It is evident that hyperglycemic conditions and the accompanying presence of dicarbonyl intermediates such as MGO tend to disturb the normal shape of RBCs. The morphological changes induced by MGO were prevented when the $\mathrm{RBC}$ were treated with $10 \mathrm{mM}$ AG 
(fig. 7C) and $200 \mu \mathrm{g}$ of MIG4 (fig. 7D). Based on these studies, it can be noted that the MIG4 could be helpful in preventing the complications caused by glycation.

Synthetic peptide (GK peptide) containing a lysine residue was incubated with ribose for $24 \mathrm{~h}$. This procedure was expected to generate peptides with advanced Maillard reaction product with dimerization through lysine-lysine cross-linking ${ }^{[22]}$. Protein crosslinking and fluorescence formation are the major end results of the Maillard reaction. On the basis of these interactions, this model system was used to evaluate the inhibitory effect of MIG4 on protein cross-linking. MIG4 exhibited substantial anticross-linking activity by decreasing fluorescence intensity, when compared to GK peptide in the presence of ribose as shown in fig. 8. At a concentration of $200 \mu \mathrm{g}$, the inhibitory effect of MIG4 was $71.29 \%$. Comparatively, AG at $10 \mathrm{mM}$ showed $84.04 \%$ inhibition. These results demonstrated the protection of MIG4 against late glycation end products. Similar studies have been reported in literature, ECG, EGCG, kaempferol and luteolin exhibited substantial anticross-linking activities. At a concentration of $100 \mu \mathrm{M}$, the inhibitory effects of EGCG and luteolin were overall $85 \%$, which was comparable to that of AG $(93.3 \%)^{[24]}$.

In conclusion, synergistic effects of MIG4 extract suggest its potential inhibitory effects at every stage of protein glycation due to the presence of different

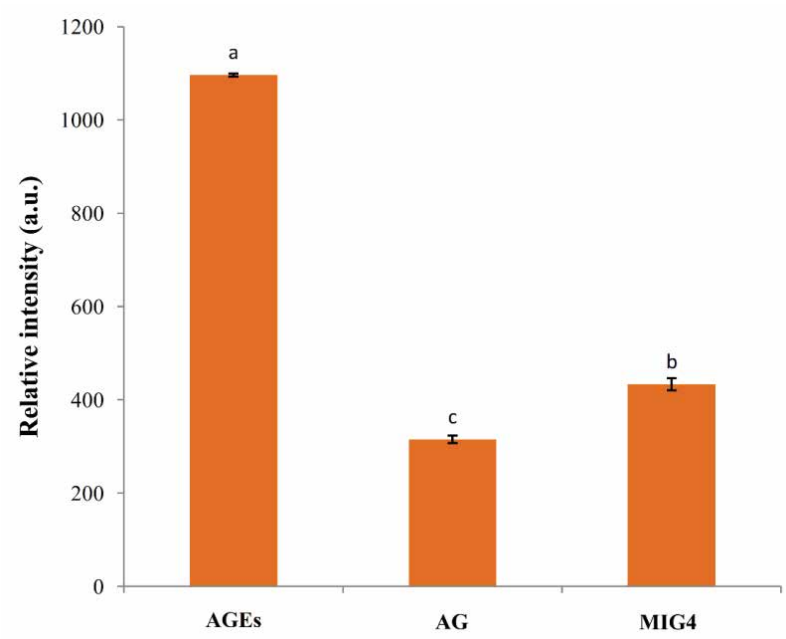

Fig. 8: Last stage of glycation by GK-peptide ribose assay GK peptide $(80 \mathrm{mg} / \mathrm{ml})$ in the presence of ribose $(0.8 \mathrm{M})$ under sterile conditions in $0.5 \mathrm{M}$ sodium phosphate buffer ( $\mathrm{pH}$ 7.4) in the absence or the presence of MIG4 at $200 \mu \mathrm{g}$ incubated at $37^{\circ}$ for $24 \mathrm{~h}$. The fluorescence of the mixture was read at 340 $\mathrm{nm}$ excitation and $420 \mathrm{~nm}$ emission wave lengths. Results are means \pm SD for $n=3$. $p<0.05$ compared with $G K+$ ribose (AGEs). Groups with different letters superscripts are significantly different $(\mathbf{p}<\mathbf{0 . 0 5})$ phytochemicals, which may interrupt the cascade of events, causing prevention of glycation. The scavenging of free radicals derived from glycation plays an important role in this reaction. This mechanism may help to provide a protective effect against hyperglycaemiamediated protein damage or may be quite useful in the treatment of pathological complications including diabetes mellitus. Further studies are needed to understand the mechanism of action. Hence, attempts are underway in assessing the potential of MIG4 as aldose reductase inhibitor in streptozotocin-induced diabetic cataract in experimental animals.

\section{Acknowledgements:}

This work received financial support from the Indian Council of Medical Research, New Delhi and DRSUGC-SAP-II, New Delhi. The first author is grateful to the Indian Council of Medical Research, New Delhi for a Senior Research Fellowship. Authors are grateful to the Institution of Excellence (IOE) and University with Potential for Excellence (UPE), University of Mysore, Mysuru for providing instrumentation facilities.

\section{Conflict of interest:}

The authors declare no conflict of interest.

\section{REFERENCES}

1. Singh VP, Bali A, Singh N, Jaggi AS. Advanced glycation end products and diabetic complications. Korean J Physiol Pharmacol 2014;18(1):1-14.

2. Bucala R, Cerami A. Advanced Glycosylation: Chemistry, biology and implications for diabetes and aging. Adv Pharmacol 1992;23:1-34.

3. Monnier VM. Nonenzymatic glycosylation, the Maillard reaction and the aging process. J Gerontol 1990;45(4):B105-11.

4. Lapolla A, Traldi P, Fedele D. Importance of measuring products of non-enzymatic glycation of proteins. Clin Biochem 2005;38(2):103-15.

5. Booth $\mathrm{AA}$, Khalifah RG, Todd $\mathrm{P}$, Hudson BG. In vitro kinetic studies of formation of antigenic advanced glycation end products (AGEs). J Biol Chem 1997;272(9):5430-7.

6. Reddy VP, Beyaz A. Inhibitors of the Maillard reaction and AGE breakers as therapeutics for multiple diseases. Drug Discov Today 2006;11(13-14):646-54.

7. Dugé de Bernonville T, Guyot S, Paulin JP, Gaucher M, Loufrani L, et al. Dihydrochalcones: Implication in resistance to oxidative stress and bioactivities against advanced glycation end-products and vasoconstriction. Phytochemistry 2010;71(4):443-52.

8. Satish A, Sairam S, Ahmed F, Urooj A. Moringa oleifera Lam.: Protease activity against blood coagulation cascade. Pharmacogn Res 2012;4(1):44

9. Satish A, Punith Kumar R, Rakshith D, Satish S, Ahmed F. Antimutagenic and antioxidant activity of Ficus benghalensis 
stem bark and Moringa oleifera root extract. Int J Chem Anal Sci 2013;4(2):45-8.

10. Urooj A, Ahmed F. Ficus racemosa and Morus indica: Emerging alternative antihyperglycemic agents. Open Conf Proc J 2013;4:59-65.

11. Satish A, Reddy PV, Sairam S, Ahmed F, Urooj A. Antioxidative effect and DNA protecting property of Moringa oleifera root extracts. J Herbs Spices Med Plants 2014;20(3):209-20.

12. Sahasrabhojaney V, Turankar AV, Motghare VM, Kumbhalkar S, Khanzode SS, Khobragade LR, et al. Hypoglycemic and Hypolipidemic Activity of Mulberry (Morus indica) in Type 2 Diabetes Patients. Indian Med Gaz 2013:8:21-7.

13. Anandan S, Kotebagilu NP, Shivanna LM, Urooj A. Inhibitory potency of c-glycosyl flavonoids from Morus sp. on advanced glycation end products. J Biol Act Prod Nat 2017;7(5):391400.

14. Deng X, Gao G, Zheng S, Li F. Qualitative and quantitative analysis of flavonoids in the leaves of Isatis indigatica Fort. by ultra-performance liquid chromatography with PDA and electrospray ionization tandem mass spectrometry detection. J Pharm Biomed Anal 2008;48(3):562-7.

15. Rahbar S, Kumar Yernini K, Scott S, Gonzales N, Lalezari I. Novel inhibitors of advanced glycation endproducts. Biochem Biophys Res Commun 1999;262(3):6510-656.

16. Prasanna G, Saraswathi NT. Aspartic acid functions as carbonyl trapper to inhibit the formation of advanced glycation end products by chemical chaperone activity. J Biomol Struct Dyn 2016;34(5):943-51.

17. Laemmli UK. Cleavage of structural proteins during the assembly of the head of bacteriophage T4. Nature 1970;227(5259):680-5.

18. LeVine H. Quantification of $\beta$-sheet amyloid fibril structures with thioflavin T. Methods Enzymol 1999;309:274-84.

19. Ashraf JM, Ansari MA, Khan HM, Alzohairy MA, Choi I. Green synthesis of silver nanoparticles and characterization of their inhibitory effects on AGEs formation using biophysical techniques. Sci Rep 2016;6(1):20414.

20. Girish TK, Prasada Rao UJ. Protein glycation and aggregation inhibitory potency of biomolecules from black gram milled by-product. J Sci Food Agric 2016;96(15):4973-83.
21. Buys AV, Van Rooy MJ, Soma P, Van Papendorp D, Lipinski B, Pretorius E. Changes in red blood cell membrane structure in type 2 diabetes: a scanning electron and atomic force microscopy study. Cardiovasc Diabetol 2013;12(1):25.

22. Nagaraj R, Shipanova, IN, Faust F. Protein cross-linking by the Maillard reaction. J Biol Chem 1996;271(32):19338-45.

23. Thornalley PJ, Langborg A, Minhas HS. Formation of glyoxal, methylglyoxal and 3-deoxyglucosone in the glycation of proteins by glucose. Biochemical J 1999;344(1):109-16.

24. $\mathrm{Wu} \mathrm{CH}$, Yen $\mathrm{GC}$. Inhibitory effect of naturally occurring flavonoids on the formation of advanced glycation endproducts. J Agric Food Chem 2005;53(8):3167-73.

25. Xi M, Hai C, Tang H, Wen A, Chen H, Liu R, et al. Antioxidant and antiglycation properties of triterpenoid saponins from Aralia taibaiensis traditionally used for treating diabetes mellitus. Redox Rep 2010;15(1):20-8.

26. Rasoanaivo P, Wright CW, Willcox ML, Gilbert B. Whole plant extracts versus single compounds for the treatment of malaria: synergy and positive interactions. Malar J 2011;10(1):S4.

27. Anwar F, Abdul Qayyum HM, Ijaz Hussain A, Iqbal S. Antioxidant activity of $100 \%$ and $80 \%$ methanol extracts from barley seeds (Hordeum vulgare L.): stabilization of sunflower oil. Grasas Aceites 2010;61(3):237-43.

28. Bonnefont-Rousselot D. Glucose and reactive oxygen species. Curr Opin Clin Nutr Metab Care 2002;5(5):561-8.

29. Khurana R, Coleman C, Ionescu-Zanetti C, Carter SA, Krishna $\mathrm{V}$, Grover RK, et al. Mechanism of thioflavin T binding to amyloid fibrils. J Struct Biol 2005;151(3):229-38.

30. FTIR analysis of protein structure. Available from: https:// www.chem.uwec.edu/chem455_s05/pages/manuals/FTIR_ of proteins.pdf.

31. Iannuzzi C, Irace G, Sirangelo I. Differential effects of glycation on protein aggregation and amyloid formation. Front Mol Biosci 2014;1:9.

32. Awasthi S, Gayathiri SK, Ramya R, Duraichelvan R, Dhason A, Saraswathi NT. Advanced glycation-modified human serum albumin evokes alterations in membrane and eryptosis in erythrocytes. Appl Biochem Biotechnol 2015;177(5):101324. 